\title{
PVA nanocomposites reinforced with cellulose nanofibers from oil palm empty fruit bunches (OPEFBs)
}

\author{
Farah Fahma ${ }^{1 *}$, Naruhito Hori², Tadahisa Iwata ${ }^{2}$, Akio Takemura ${ }^{2 *}$ \\ ${ }^{1}$ Department of Agroindustrial Technology, Faculty of Agricultural Engineering and Technology, Bogor Agricultural University, Bogor, Indonesia, \\ ${ }^{2}$ Department of Biomaterial Sciences, Graduate School of Agricultural and Life Sciences, The University of Tokyo, Tokyo, Japan
}

\section{A B S TR A C T}

The objective of this study is to investigate the effect of sonication time on the morphology and properties of poly(vinyl alcohol) (PVA)cellulose nanocomposite. PVA nanocomposite films were prepared by reinforcement of cellulose nanofibers into a PVA matrix with different sonication time. Two series of PVA films with and without cellulose nanofibers were prepared by varying the sonication time. The effect of sonication time on the morphology and properties of the films were investigated by SEM, FTIR and Raman spectroscopy, TGA, and tensile test. The results showed that the mechanical property of the nanocomposite films increased with increasing sonication time until 9 min.

Keywords: Sonication time; PVA; Nanofibers; Nanocomposite

\section{INTRODUCTION}

Lately, numerous efforts have been focused on using materials from renewable resources for nanocomposite reinforcement. Cellulose, which is known due its availability and excellent properties, especially when in the form of nanofibers, has attracted much attention from many researchers. The addition of cellulose nanofibers in polymer matrix is a great interest to replace the nanofiller from non-renewable materials.

In Indonesia, the oil palm industries generate abundant amount of oil palm empty fruit bunches (OPEFBs) in millions of tons per year which causes serious adverse environmental impacts. The utilization of OPEFBs has attracted a great deal of interest as a reinforcing agent in composite materials (Fahma et al., 2010).

In our previous work, the cellulose nanofibers were successfully isolated and characterized from oil palm empty-fruit-bunch (OPEFB) (Fahma et al., 2010) and coconut husk (Fahma et al, 2011). Nanofibers from OPEFB demonstrated that its degree of polymerization (DP) value and thermal stability were higher than that of from coconut husk. Meanwhile, other properties were similar for each other.

Hydrogen bonding between cellulose chains in cellulose nanofibers causes agglomeration or entanglement of nanofibers in the polymer matrix. Therefore, to reduce the interaction between hydroxyl groups, the cellulose nanofibers are maintained in the form of suspension in water. Thus the use of cellulose nanofibers without modification for the composites is limited to water soluble polymer (Wang and Sain, 2007).

Poly(vinyl alcohol) (PVA) is a water soluble polymer which its use is widespread in many areas of industrial applications such as textiles, paints, and adhesive industries and it is also a very promising candidate for the preparation of biodegradable plastics. This is because of its biodegradability, biocompatibility, chemical resistance and excellent physical properties (Nwufo et al. 1984; Sapalidis et al., 2007; Chen et al., 2008). The hydroxyl

\footnotetext{
*Corresponding author:

Farah Fahma, Department of Agroindustrial Technology, Faculty of Agricultural Engineering and Technology, Bogor Agricultural University, Bogor, Indonesia. E-mail: farah_fahma@yahoo.com

Akio Takemura, Department of Biomaterial Sciences, Graduate School of Agricultural and Life Sciences, The University of Tokyo, Tokyo, Japan. E-mail: akiot@mail.ecc.u-tokyo.ac.jp
} 
groups on PVA matrix are expected to interact with the hydrophilic surfaces of the cellulose nanofibers, leading to strong hydrogen bonding. PVA-based fibers have been considered as an attractive choice in tissue scaffolding, filtration materials, membranes, optics, protective clothing, enzyme immobilization, drug release, and so on (Peresin et al., 2010).

Gea et al. (2010) prepared bacterial cellulose-PVA nanocomposites by an in-situ process. Meanwhile, Lu et al. (2008) prepared PVA composites reinforced with microfibrillated cellulose (MFC). The mixing process between MFC and PVA solution was done by 1 min of sonication time and followed by stirring for $24 \mathrm{~h}$ to let the polymer penetrate into the cellulose network. The tensile strength and modulus increased with increasing the MCC content up to $10 \mathrm{wt} \%$ and then tends to level off at higher MFC content.

Cheng et al. (2009) prepared PVA nanocomposites reinforced with cellulose fibrils (regenerated cellulose fibers, pure cellulose fibers, and MCC). The mixture solution of PVA nanocomposites was sonicated for 1 min with 50\% power level. Adding more than $6 \mathrm{wt} \%$ cellulose fibrils did not increase more strength and modulus.

Chen et al. (2008) prepared PVA-pea starch nanocrystals composites by adding glycerol. The mixture solution was stirred for $30 \mathrm{~min}$ at $100^{\circ} \mathrm{C}$. The tensile strength and elongation at break of nanocomposite films with 5 and 10 $\mathrm{wt} \%$ of nanocrystals were slightly higher than that of neat PVA film. With an increase of cellulose nanocrystals, the tensile strength and elongation at break of nanocomposite films decreased and become lower than that of neat PVA film.

Cho and Park (2011) prepared PVA nanocomposites reinforced with nanocellulose isolated by sulfuric acid hydrolysis using commercial microcrystalline cellulose (MCC). The MCC added in PVA matrix was 1, 3, 5 , and $7 \mathrm{wt} \%$ loadings. The PVA nanocellulose suspension was further stirred mechanically for $2 \mathrm{~h}$ and sonicated for $10 \mathrm{~min}$. The tensile strength and modulus increased with an increase in the nanocellulose content up to $5 \mathrm{wt} \%$ followed by leveling off at higher nanocellulose content.

Lee et al. (2009) prepared PVA composite films reinforced with nanocellulose obtained by acid hydrolysis of MCC at different hydrobromic acid ( $\mathrm{HBr}$ ) concentrations. The nanocelluloses added to the PVA solution were 1,3 , and 5 $\mathrm{wt} \%$. The mixture was stirred at $80^{\circ} \mathrm{C}$ for $2 \mathrm{~h}$ and followed by ultrasonication for $1 \mathrm{~h}$. The nanocellulose loading of 3 and $5 \mathrm{wt} \%$ to PVA matrix gradually decreased the tensile strength.
Sonication is often used in the dispersion of nanoparticles into polymer matrix. The basic principle of the enhanced dispersion is the ultrahigh shear rate attained during cavitation events (Huang et al., 2009).

Although many works have been done on the PVA nanocomposites reinforced with cellulose nanofibers, how long the sonication treatment effectively help dispersing nanofibers into the matrix is not clear. Therefore the objective of this study is to investigate the effect of sonication time on the morphology and properties of PVA cellulose nanocomposite.

\section{MATERIALS AND METHODS}

\section{Materials}

OPEFBs supplied by PT Perkebunan Nusantara VIII Kertajaya, Lebak, Indonesia were used for obtaining nanofibers. Sulfuric acid (95\%), Poly(vinyl alcohol) (PVA, DP 2000) and other chemicals such as ethanol, benzene, sodium chlorite, acetic acid, and potassium hydroxide were supplied by WAKO Pure Chemical Industries Ltd., Japan.

\section{Preparation of nanofiber suspension}

Cellulose nanofibers from OPEFB were prepared as described in our previous work (Fahma et al., 2010, 2011). The cellulose fibers were hydrolyzed in sulfuric acid solution $(64 \mathrm{wt} \%)$ under strong agitation at $45^{\circ} \mathrm{C}$ for $60 \mathrm{~min}$. The reaction of hydrolysis was stopped by adding cold water. Subsequently, the suspension was washed by deionized water with centrifugation several times, then dialyzed and sonicated for $20 \mathrm{~min}$ to disperse nanofibers in water. The suspensions were fractionated by centrifugation at $18,000 \mathrm{rpm}$ for $10 \mathrm{~min}$ to get high dispersed nanofibers. Finally, the high dispersed nanofiber suspension was stored in a refrigerator before nanocomposite preparation.

\section{PVA cellulose nanocomposite preparation}

PVA cellulose nanocomposites with $5 \mathrm{wt} . \%$ of nanofibers were prepared as follows. PVA water solution $(10 \mathrm{wt} \%)$ and the high dispersed nanofiber suspension were mixed and stirred manually and then dispersed by using an ultrasonic homogenizer for $0,1,3,5,7,9$, and 20 min with $50 \%$ power level at $19.5 \mathrm{kHz}$ and $300 \mathrm{~W}$ output power $(7 \mathrm{~mm}$ probe tip diameter, US-300T, Nissei, Japan). The mixtures were degassed by allowing them with caps at room temperature overnight. Subsequently, the mixtures were cast in Petri dishes and dried at room temperature for several days. The resulting films were coded as PVA-NF-tst, where $t$ stands for the length of sonication time. Another series of PVA water solutions without cellulose nanofiberswere prepared at the same treatment as a comparison and coded as PVA-tst, where $t$ stands for the length of sonication 
time. The thickness of all samples (PVA-NF-tst and PVAtst) was around $237 \pm 23.6 \mu \mathrm{m}$. The samples were kept in adesiccator before analyses were carried out.

\section{Characterizations of PVA cellulose nanocomposites}

The morphology of nanocomposites was evaluated by employing a scanning electron microscope (SEM, JEO S4800) operating at $1 \mathrm{kV}$. Thin nanocomposite films for fourier transform infrared spectroscopy (FTIR) analysis were prepared by spin coater $500 \mathrm{rpm}$. FTIR spectra were recorded using a Nicolet MAGNA-IR 860 spectrometer in absorbance mode with resolution $4 \mathrm{~cm}^{-1}$; 64 scans were accumulated for each sample. Raman spectra were recorded using NXR FT-Raman module (ThermoScientific, USA) and 1000 scans were accumulated for each sample. Thermogravimetric analysis (TGA) was carried out using a ThermoPlus TG 8120 instrument. The thermograms were acquired between 30 and $500^{\circ} \mathrm{C}$ at heating rate of $10 \mathrm{~K} \mathrm{~min}^{-1}$, with nitrogen as purge gas at flow rate of $110 \mathrm{~mL} \mathrm{~min}^{-1}$. An empty pan was used as reference. The tensile tests for all samples were performed using EZ Test machine (Shimadzu, Japan). The specimens were $3 \mathrm{~mm}$ wide, $10 \mathrm{~mm}$ long and around $237 \pm 23.6 \mu \mathrm{m}$ thick. Three measurements were done to characterize each sample.

\section{RESULTS AND DISCUSSION}

In this research, two PVA films were prepared, PVA film with and without cellulose nanofibers. There are intended to investigate the effect of sonication time on the properties of PVA-nanocomposites.

\section{Morphology of the nanocomposites}

Generally, all images show that with an increase in sonication time cellulose nanofibers dispersed well in the PVA matrix. The transparency of the resulting PVA cellulose nanofibers with different sonication time films confirms the homogeneous dispersion of cellulose nanofibers in PVA.

The cellulose nanofibers from OPEFB used to prepare PVA nanocomposite had average thickness around 2-3 nm (Fig. 1). The nanofibers presented in Fig. 1 mostly have long fibers ca. 1-2 $\mu \mathrm{m}$ of length.It was very difficult to observe the dispersion of small size nanofibers in the PVA matrix by SEM (scanning electron microscope) with high magnification due to blurry images. Fig. 2 shows SEM micrographs of PVA nanocomposites at $10 \mathrm{~K}$ magnification. The SEM micrograph of PVA-NF-0ST shows that cellulose nanofibers did not dispersed well in PVA matrix due to appearance of the two parts (A and B) were not mixed. Part A was cellulose nanofibers and part B was PVA matrix. From Fig. 2 we can see the difference

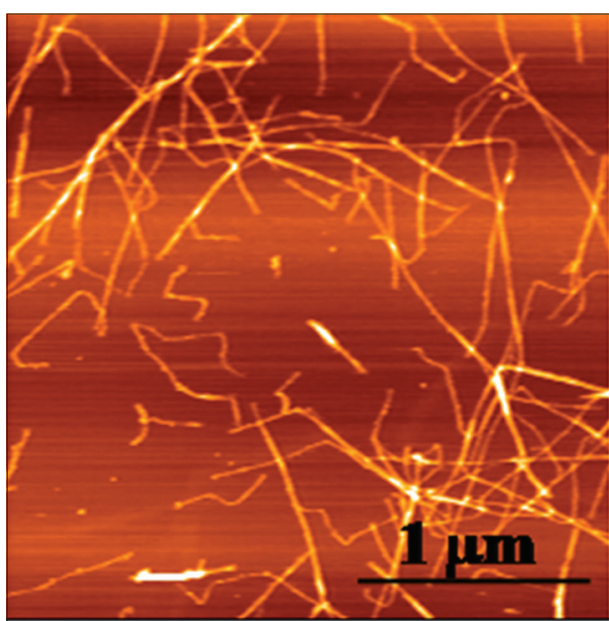

Fig 1. AFM image of nanofibers from OPEFBs.

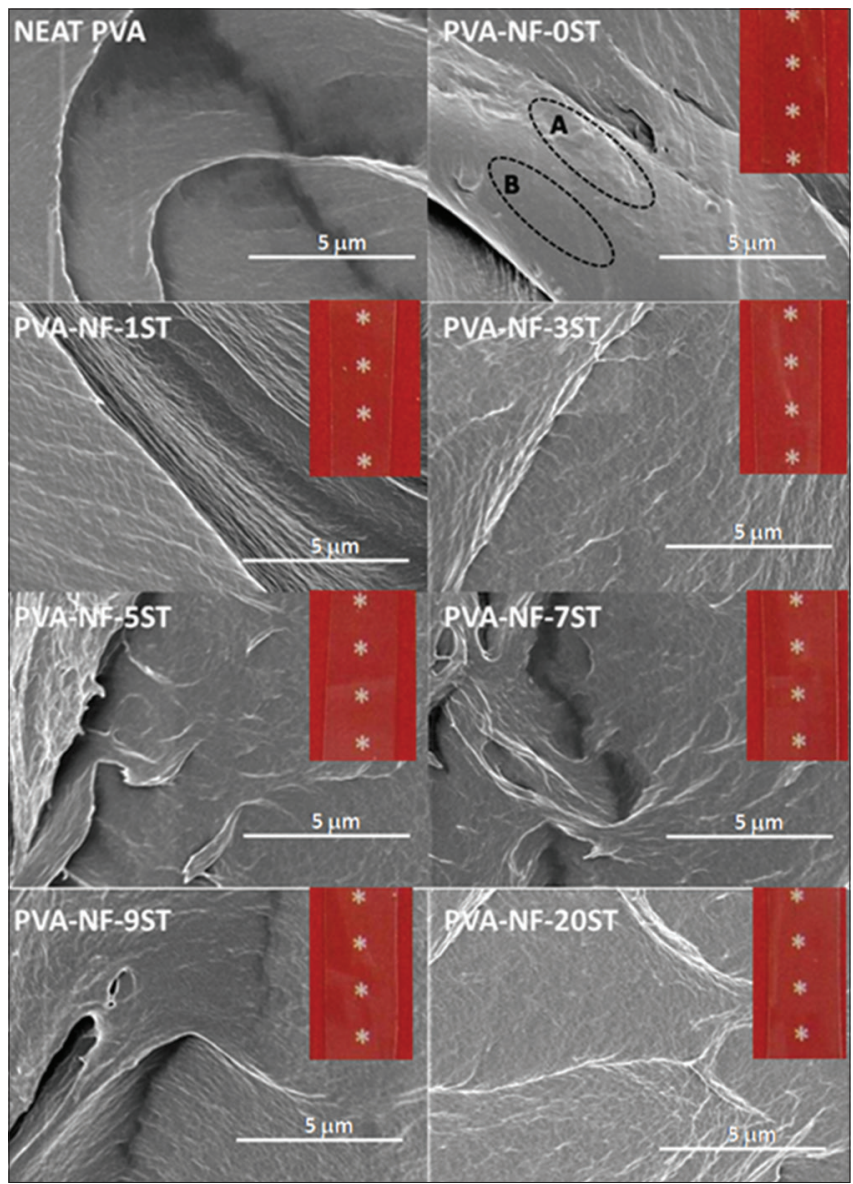

Fig 2. SEM micrographs of PVA nanocomposites.

between the morphology of fracture surfaces of PVA without and with cellulose nanofibers. Fracture surface of PVA without cellulose (neat PVA) seems smooth without like-fine-fibers. Meanwhile, the fracture surface of the PVAs with cellulose nanofibers with 1-20 min sonication treatment seems to transform into a surface with likefine-fibers. 


\section{FTIR and Raman spectra}

FTIR and Raman spectroscopy were used to observe the changes in chemical composition due to different sonication time on the neat PVA and its nanocomposites. The FTIR spectra of neat PVA and its nanocomposites with different sonication time are shown in Fig. 3.

Fig. 3A shows the similarity of the spectra of all neat PVA films even they were sonicated with different time. The peaks for all neat PVA (Fig. 3A) at around $3330 \mathrm{~cm}^{-1}$ represent hydrogen-bound hydroxyl groups between polymer chains. As cellulose nanofibers were added to PVA matrix, the location of the hydroxyl bands of all nanocomposites with different sonication treatment shifted to $3336 \mathrm{~cm}^{-1}$, representing the interaction of the components. The peak for cellulose nanofibers located at $3345 \mathrm{~cm}^{-1}$ also corresponds to hydroxyl groups. The location of shifted peaks showed the similarity of all peaks for PVA nanocomposites with increasing sonication time.
The peaks at 1440 and $1093 \mathrm{~cm}^{-1}$ are attributed to acetate $\mathrm{C}=\mathrm{O}$ stretching and acetate $\mathrm{C}-\mathrm{O}$ bending, respectively. The shoulder peak at $1060 \mathrm{~cm}^{-1}$ which are attributed to C-O stretching only could be detected in PVA nanocomposites (Fig. 3B). The intensity of these peaks are shown just slightly because of the small content of nanofiber in PVA matrix $(5 \mathrm{wt} \%)$. Location of all absorbance bands shows no obvious changes for all PVA nanocomposites as sonication time increased. These results indicate that there was no change of the FTIR spectra even after 20 min sonication time.

The Raman spectra in Fig. 3 (right) show the similarity of the spectra of all neat PVA and their nanocomposites even those have different sonication time. All peaks appearing in Raman spectra mostly seem from the vibrations of PVA molecules. The peaks at around $2912 \mathrm{~cm}^{-1}$ are assigned to the stretching vibrations of $\mathrm{CH}_{2}$ and $\mathrm{CH}$, while the other peaks at around $1440 \mathrm{~cm}^{-1}$ are assigned to the stretching vibrations of $\mathrm{CH}$ and $\mathrm{OH}$ in the PVA molecules (Uddin, et al. 2011). The sonication time seems to influence

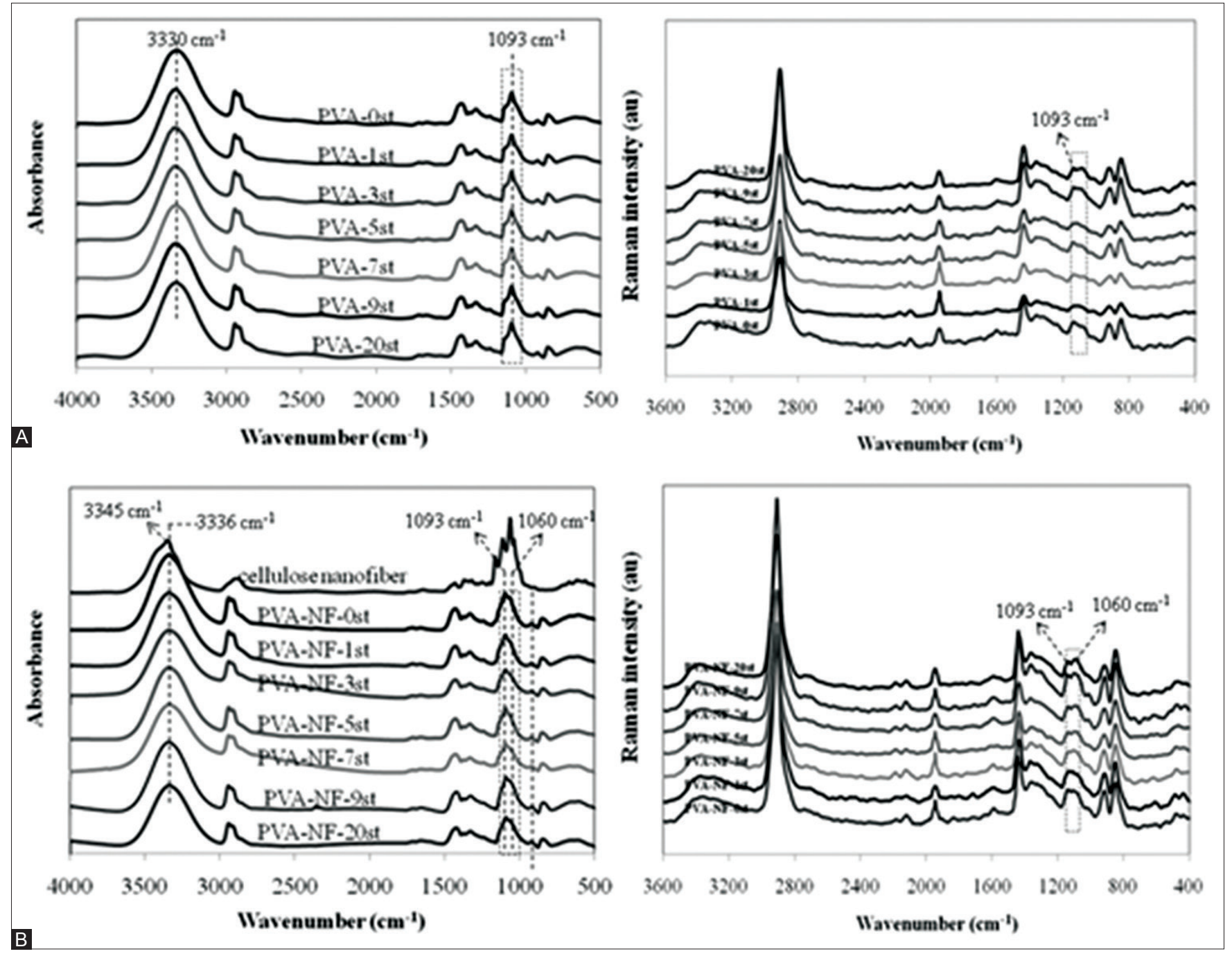

Fig 3. FTIR ad Raman spectra of neat PVA (A) and its nanocomposites (B) with different sonication time. 
the broad peak for PVA nanocomposite at around $1093-1060 \mathrm{~cm}^{-1}$ whereas with increasing sonication time these broad peaks appear to be two peaks with low intensity, i.e. at $1093 \mathrm{~cm}^{-1}$ and $1060 \mathrm{~cm}^{-1}$. Meanwhile for neat PVA these peaks seem to be still single broad peak, $1093 \mathrm{~cm}^{-1}$.

\section{Thermal properties}

TGA measurements were carried out in order to understand the thermal stability of neat PVA and its nanocomposites. Fig. 4 shows that all PVA-cellulose nanocomposites start to degrade at higher temperatures than those of neat PVA. This indicates that the addition of cellulose nanofiber can improve thermal stability of PVA, thus confirming the enhanced thermal stability due to a strong hydrogen bonding between the hydroxyl groups of nanofibers and the PVA matrix (Lee et al., 2009). The 0-9 min of sonication time did not affect the thermal stability of PVA nanocomposites while with $20 \mathrm{~min}$

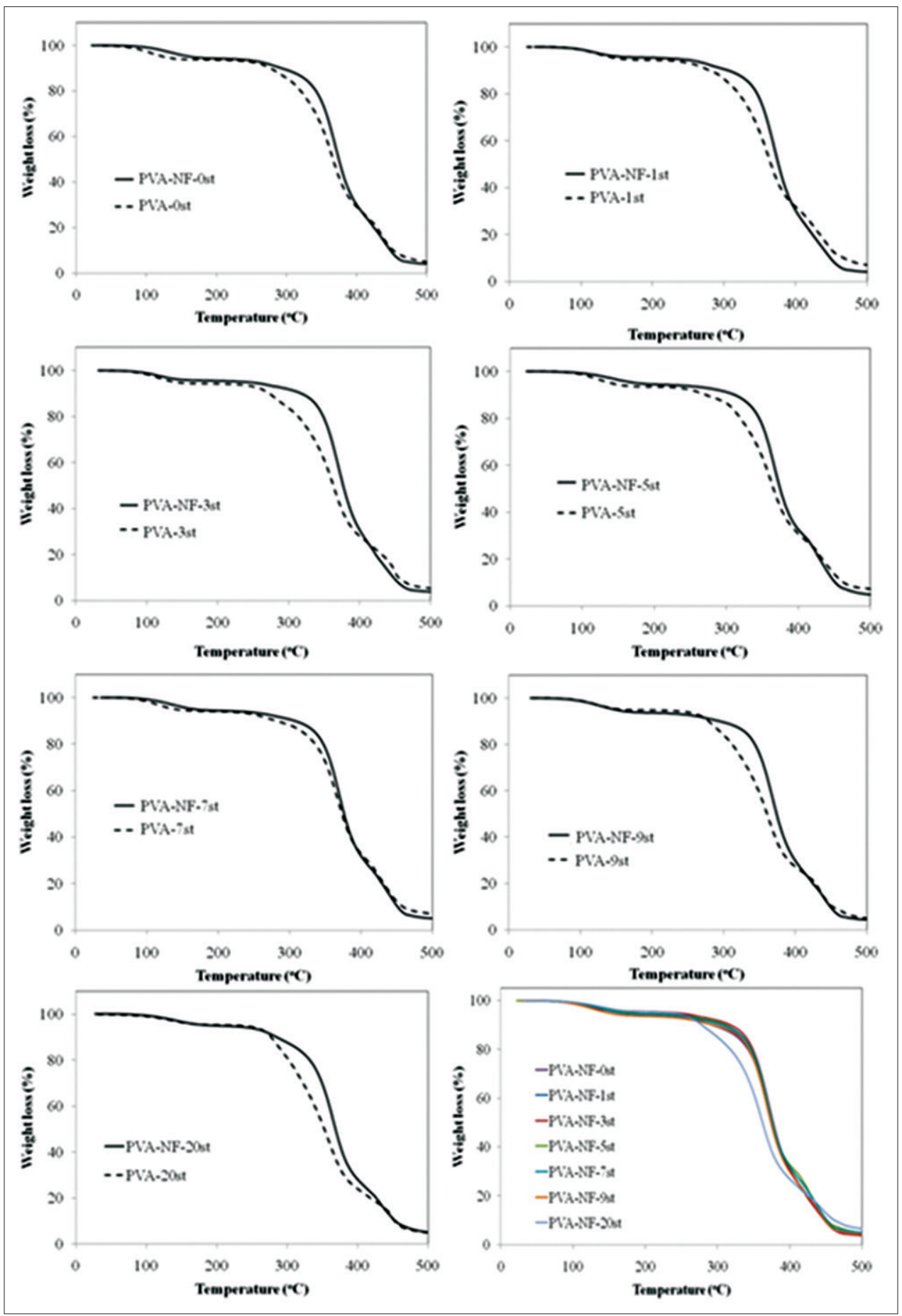

Fig 4. TGA curves of neat PVA and its nanocomposites with different sonication time. 
sonication time the degradation temperature shifted to lower temperatures.

Both TGA thermograms of all neat PVA and their nanocomposites show similar patterns, i.e. three main weight loss regions. The first region $\left(75-160^{\circ} \mathrm{C}\right)$ is due to the evaporation of water, and the weight loss of the film in these ranges is about $5-10 \mathrm{wt} \%$. The second transition region $\left(260-390^{\circ} \mathrm{C}\right)$ is due to the structural degradation of PVA nanocomposite films and the total of weight loss in this range was approximately $70 \mathrm{wt} \%$. The third region occurred above $390^{\circ} \mathrm{C}$, probably due to the cleavage backbone of PVA nanocomposite films or the decomposition of carbonaceous matter. The total weight loss in this range was more than $95 \mathrm{wt} \%$ at $500^{\circ} \mathrm{C}$ (Lee et al., 2009; Lu et al., 2009).

\section{Mechanical properties of the nanocomposites}

The tensile strength and modulus of neat PVA and its nanocomposites are shown in Fig. 5. With increasing sonication time all neat PVA showed that tensile strength and modulus tended to be constant.

The tensile strength of the PVA reinforced with cellulose nanofibers showed lower for $0-1$ min sonication time and higher for 3-9 min sonication time than that of the neat PVA. This might be for less than 3 min sonication time, there were no enough energy given to polymer/cellulose nanofibers mixture, the nanofibers could not escape from the restraining force within the nanofibers clusters; thus the dispersion was limited. From 3 to 9 min sonication time, the tensile strength of nanocomposite films increased and decreased when nanocomposite mixture was sonicated for $20 \mathrm{~min}$. This might be too much energy was given to the nanofibers to move around, then the frequency of collision between each single nanofiber will be increased. Hence, the dispersion mechanism may be adversely affected with too much energy given to the nanofibers. Therefore, an optimum sonicating time must be achieved in order to have the maximum dispersion ability (Lam et al., 2005; Kabir et al., 2007).

The increase of tensile strength of PVA matrix after adding cellulose nanofibers indicated that good dispersion of nanofibers into PVA matrix and interfacial adhesion between PVA matrix and nanofibers were achieved. PVA and cellulose nanofibers showed good miscibility due to the mutual ability of PVA and cellulose nanofibers to form intrainter molecular hydrogen bonds between hydroxyl groups.

The tensile modulus of PVA nanocomposites tended to be lower than that of the neat PVA when the sonication time was less than 9 min. However, after 9 and 20 min sonication time the PVA nanocomposites had higher tensile modulus than that of neat PVA.

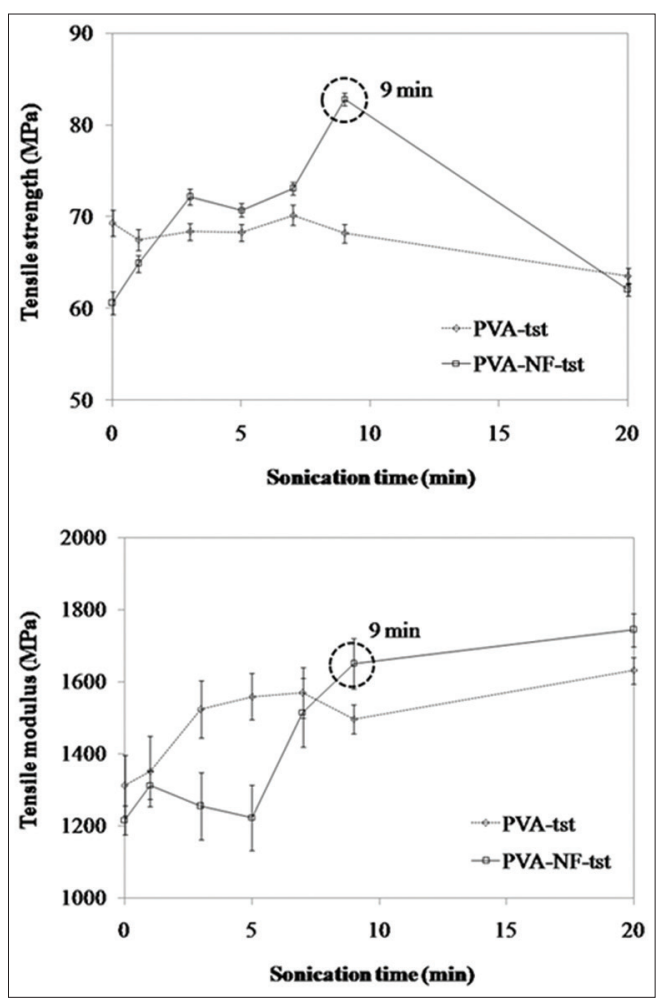

Fig 5. Tensile strength and modulus of neat PVA and its nanocomposites with different sonication time.

From some research described in the part of introduction, it is supposed that the mixing methods between the cellulose nanofibers and PVA solution significantly affect the properties of the resulting nanocomposites. Even PVA is water soluble and cellulose nanofibers are dispersed well in water. Lee et al. (2009) reported that with $1 \mathrm{~h}$ of sonication time the addition of 3 and $5 \mathrm{wt} \%$ nanocellulose gradually decreased the tensile strength of PVA matrix. This might be with $1 \mathrm{~h}$ of sonication too much energy was given. The different thing was reported by Cho and Park (2011). With 10 min sonication time, the tensile strength and modulus of PVA nanocomposite with $5 \mathrm{wt} \%$ cellulose nanofibers showed higher values than those of neat PVA. It is supposed that $10 \mathrm{~min}$ sonication time gave enough energy for cellulose nanofibers to disperse into PVA matrix.

The addition of cellulose nanofibers improved the mechanical and thermal properties of PVA nanocomposites. For the use of these nanocomposites in filtration and membrane application, the stability in presence of water needs to investigate.

\section{CONCLUSION}

The mechanical properties showed that there was optimum value of tensile strength with increasing sonication time. The tensile strength of PVA reinforced with cellulose 
nanofibers were improved with increasing sonication time from $3 \mathrm{~min}$ to $9 \mathrm{~min}$ and then decreased when the sonication time was $20 \mathrm{~min}$. Sonication time within 9 min was able to maintain the thermal stability of PVA nanocomposites. Even though the thermal stability of the nanocomposite shifted to lower temperature after $20 \mathrm{~min}$ sonication time.

\section{Conflicts of interest}

The authors declare that there is no conflict of interest regarding the publication of this manuscript. This manuscript has not been published and is not going to be considered for publication elsewhere. The authors certify that neither the manuscript nor its main contents have already been published or submitted for publication in another journal.

\section{Authors contributions}

All the authors contributed equally to the writing of this paper. They were also involved in the overall work of experiments

\section{REFERENCES}

Chen, L., X. Cao, P. R. Chang and M. A. Huneault. 2008. Comparative study on the films of poly(vinyl alcohol)/pea starch nanocrystals and poly(vinyl alcohol)/native pea starch. Carbohydr. Polym. 73: 8-17.

Cheng, Q., S. Wang and T. G. Rials. 2009. Poly(vinyl alcohol) nanocomposites reinforced with cellulose fibrils isolated by high intensity ultrasonication. Compos. Part A. 40: 218-224.

Cho, M. J. and B. D. Park. 2011. Tensile and thermal properties of nanocellulose-reinforced poly(vinyl alcohol) nanocomposites. J. Ind. Eng. Chem. 17: 36-40.

Fahma, F., S. Iwamoto, N. Hori, T. Iwata and A. Takemura. 2010. Isolation, preparation, and characterization of nanofibers from oil palm empty-fruit-bunch (OPEFB). Cellulose. 17: 977-985.

Fahma, F., S. Iwamoto, N. Hori, T. Iwata and A. Takemura. 2011. Effect of pre-acid-hydrolysis treatment on morphology and properties of cellulose nanowhiskers from coconut husk. Cellulose. 18: 443-450.

Gea, S., E. Bilotti, C. T. Reynolds, N. Soykeabkeaw and T. Peijs. 2010. Bacterial cellulose-poly (vinyl alcohol) nanocomposites prepared by an in-situ process. Mater. Lett. 64: 901-904.

Huang, Y. Y., T. P. J. Knowles and E. M. Terentjev. 2009. Strength of nanotubes, filaments, and nanowires from sonication-induced scission. Adv. Mater. 21: 38-39.

Kabir, M. E., M. C. Saha and S. Jeelani. 2007. Effect of ultrasound sonication in carbon nanofibers/polyurethane foam composite. Mater. Sci. Eng. A. 459: 111-116.

Lam, C., K. Lau, H. Cheung and H. Ling. 2005. Effect of ultrasound sonication in nanoclay clusters of nanoclay/epoxy composites. Mater. Lett. 59: 1369-1372.

Lee, S. Y., D. J. Mohan, I. A. Kang, G. H. Doh, S. Lee and S. O. Han. 2009. Nanocellulose reinforced PVA composite films: Effects of acid treatment and filler loading. Fiber. Polym. 10:77-82.

Lu, J., T. Wang and L. T. Drzal. 2008. Preparation and properties of microfibrillated cellulose polyvinyl alcohol composite materials. Compos. Part A. 39: 738-746.

Nwufo, B. T., G. J. L. Griffin and K. I. Ekpenyong. 1984. Extrusion of strarch-extended water-soluble polyvinyl alcohol. Ind. Eng. Chem. Prod. Res. Dev. 23: 594-595.

Peresin, M. S., Y. Habibi, J. O. Zoppe, J. J. Pawlak and O. J. Rojas. 2010. Nanofiber composites of polyvinyl alcohol and cellulose nanocrystals: Manufacture and characterization. Biomacromolecules. 11: 674-681.

Sapalidis, A. A., F. K. Katsaros, G. E. Romanos, N. K. Kakizis and N. K. Kanellopoulos. 2007. Preparation and characterization of novel poly-(vinyl alcohol)-Zostera flakes composites for packaging applications. Compos. Part B. 38: 398-404.

Uddin, A. J., J. Araki and Y. Gotoh. 2011. Characterization of the poly (vinyl alcohol)/cellulose whisker gel spun fibers. Compos. Part A. 42: 741-747.

Wang, B. and Sain, M. 2007. Dispersion of soybean stock-based nanofiber in a plastic matrix. Polym. Int. 56: 538-546. 